\title{
Systems Biology-Based Diagnostic Principles as Pillars of the Bridge between Chinese and Western Medicine
}

Authors

Affiliations
Jan van der Greef ${ }^{1,2,3,5 *}$, Herman van Wietmarschen ${ }^{1,3 *}$, Jan Schroën ${ }^{1 *}$, Mei Wang ${ }^{1,5}$, Thomas Hankemeier ${ }^{1,3}$, Guowang $\mathbf{X u}^{1,4}$

The affiliations are listed at the end of the article

\author{
Key words \\ - personalized medicine \\ - Chinese medicine \\ - diagnosis \\ - systems biology \\ - rheumatoid arthritis \\ - metabolomics \\ - phenotyping \\ - targeted care
}

\begin{abstract}
$\nabla$

Innovative systems approaches to develop medicine and health care are emerging from the integration of Chinese and Western medicine strategies, philosophies and practices. The two medical systems are highly complementary as the reductionist aspects of Western medicine are favourable in acute disease situations and the holistic aspects of Chinese medicine offer more opportunities in chronic conditions and for prevention. In this article we argue that diagnosis plays a key role in building the bridge between Chinese and Western medicine. Recent advances in the study
\end{abstract}

\section{Introduction}

$\nabla$

The health care system in Western societies and one of its pillars, modern medicine, are increasingly under debate. The increasing costs of health care are reaching the level where it will not be sustainable in the near future. Recent estimates for the US health care system show an increase from $17 \%$ of the gross domestic product (GDP) in 2009 to over $20 \%$ of the GDP in 2018, when the total cost will reach US \$ 4.35 trillion [1]. At the same time, hopes for developing medicines that are more effective are fading away, since the pharmaceutical industry produces fewer new chemical entities (NCE's) that reach the market every year.

In this era, crucial global issues have surfaced in various domains, including the financial, ecological, political, educational, religious/spiritual, and social realms. Mechanisms that previously functioned effectively must be replaced by new approaches. These challenges are not local, but global, and system thinking will be mandatory in the

\footnotetext{
* Each of these authors has equally contributed to this paper.
}

of health, healing, placebo effects and patientphysician interactions will be discussed pointing out the development of a system-based diagnosis. Especially, a system biology-based diagnosis can be used to capture phenotype information, leading towards a scientific basis for a more refined patient characterization, new diagnostic tools and personalized heath strategies. Subtyping of rheumatoid arthritis patients based on Chinese diagnostic principles is discussed as an example. New insights from this process of integrating Western and Chinese medicine will pave the way for a patient-centred health care ecosystem.
Correspondence Jan van der Greef TNO Quality of Life P. O. Box 360 3700 AJ Zeist The Netherlands Phone: + 31306944843 Fax: + 31306944040 jan.vandergreef@tno.nl future. In such times of change, one must reflect and focus on the driving force of the system that needs to evolve. In health care, there must be a shift from an emphasis on economics, technological developments and political agendas back to a patient-centred health care environment.

In health care, the paradigm of system thinking has emerged as systems biology. It is limiting to develop interventions on how a single compound interacts with a single target that is linked to a specific symptom. This "one-drug-fits-all" paradigm has shifted to an idea that patients require personalized medicines. Some successful examples have been used to treat cancer, including Herceptin ${ }^{\circledR}$ (trastuzumab), Gleevec ${ }^{\circledR}$ (imatinib mesylaat) and Iressa ${ }^{\circledR}$ (gefitinib). However, a thorough systems approach has not yet been applied to design new drugs. Moreover, the patient is not yet seen as a unique individual. Treatments focus on a disease or a disease phenotype rather than the person, even though effective treatments rely on a proper systems diagnosis.

In this paper, we describe how the global nature of challenges must be recognized to develop global solutions. These solutions must integrate diverse concepts and intuition to bring about a 
new understanding. Our discussion begins with a reflection on how Chinese and Western Medicine evolved over time. In the second step, we consider the diagnosis as the basis in each medical system, and then we consider new ways of merging knowledge from both systems ( $\bullet$ Fig. 1). This information is used to provide a deeper insight into personalized health diagnostics, and we envision the development of a future system. This type of process requires multiple steps. As an example, we use rheumatoid arthritis (RA) to describe how to diagnose and ultimately establish a personalized health care ecosystem.

\section{Bridging Chinese and Western Medicine: A Philosophical and Historical View \\ $\nabla$}

Medicine has been practised since prehistoric times, and over time, a variety of medical traditions developed. In ancient Greece, biological theories were focused on solving one problem, that of the relationship between the "one" and the "many." This union between the many phenomena formed nature, which was rationalized in the theory of the four humours. In Hippocrates' and Galen's times, disease was still perceived as a natural or physiological process, and diagnoses were made by knowing this story of transformation [3]. Centuries later, Paracelsus developed a new concept of disease based on pathological, anatomic and metabolic changes, which resulted in a new diagnostic practice that was based on the classification of diseases [4]. Thus, the perspective of disease as a process and a relation between the one and the many shifted towards viewing disease as a structure located in an individual.

Chinese medicine developed in a Taoist, Buddhist and Confucian culture. Many of the key aspects of Chinese medicine are explored in one of the oldest classical Chinese texts, the Yi Jing, a book that is still considered essential when studying and practising Chinese medicine today [5]. The Yi Jing presents what we would today refer to as a systems view of life, health and society. This view is represented beautifully in the Tai Ji symbol (๑ Fig. 2). In the Tai Ji symbol, Yin (female, earth) and Yang (male, heaven) grasp each other but are also present within each other, as represented by the small dots. The Tai Ji symbol is the oldest known example of fractal thinking because the small dot of Yin within Yang is actually another Tai Ji symbol [6]. This relates to the view expressed in the Yi Jing that the world inside the body is a reflection of and thus connected with the world outside the body ( Fig. 3). In the classical text on Chinese medicine Neijing Suwen, Qi Bo answered the Yellow Emperor saying, "The most important element in clinical diagnosis is to know the relationships between heaven, earth and humankind" [7]. Both the body and the world are complex, dynamic systems with fractal relationships to one another. In a healthy situation, Yin and Yang are in balance. This concept of balance, movement and connection was developed further in the Zang Fu (organ theory) and Five Phases theories.

Although the ancient Greek and Chinese ideas on health and medicine show many similarities, the variations in cultures and philosophies gave rise to the differences between the medical systems that we perceive today [2]. For instance, during the 17 th century, Descartes proposed that there was a division between the mind and body, and this idea had an enormous impact on Western science and medicine. Western science evolved into an objective methodology where the scientist explained and analyzed, but aimed not to be part of the studied object. Subjects related to matter became the province of science, while subjects

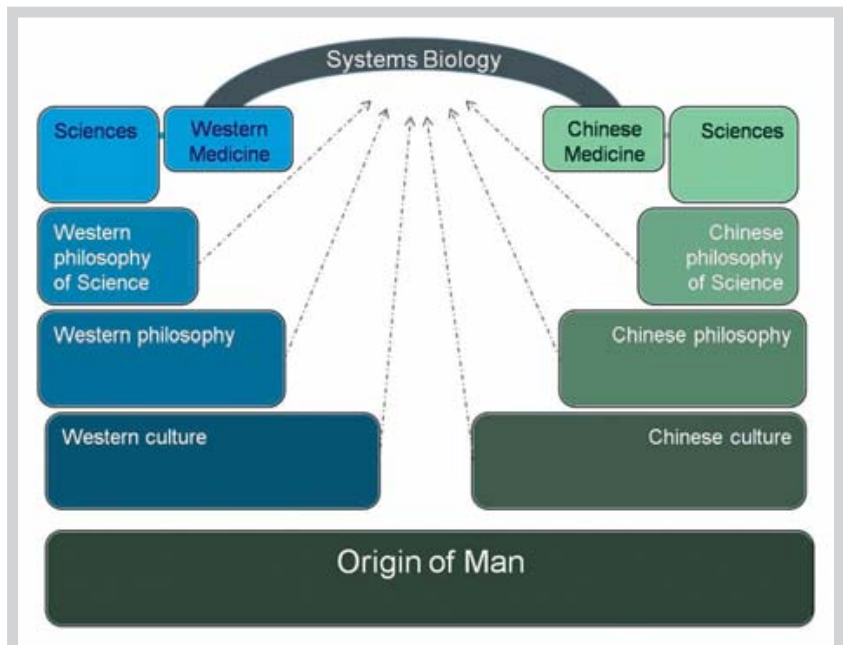

Fig. 1 Systems biology as a bridge between Chinese and Western medicine. As migration moved populations apart in space and time, the Western and Chinese cultures evolve separately, giving rise to specific cultural philosophies. These philosophies gave rise to philosophies of science, which led to types of science. This evolution allowed different types of science to exist alongside one another [2].

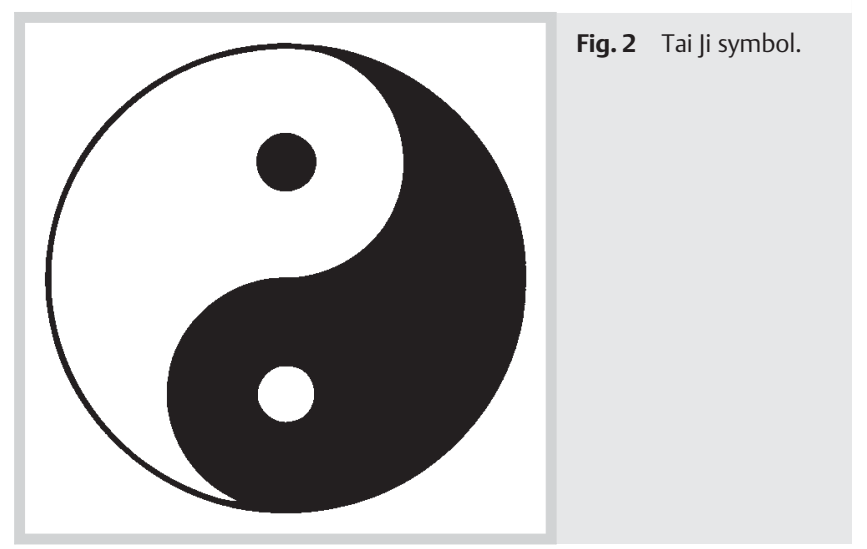

related to the mind and spirit entered the domain of religion [8]. Physicians were educated to treat patients as material systems, or physical machines, with medications and interventions for physical problems [9]. Illnesses and diseases were perceived as being located in the patient, rather than being embedded in networks, relationships, the environment and culture.

As a result, the modern Western scientific method was used to gather a tremendous amount of knowledge about anatomy, physiology, cells, genes, proteins, metabolites and more. Over the last decades, various thinkers, who were interested in how all of these aspects worked together, began combining theories from many disciplines into a systems view on life. One intriguing example is the Santiago theory of cognition in which the mindbody dualism is overcome by identifying cognition, the process of knowing, with the process of life and the body as the material structure to accommodate this process. Mental activity or the mind is an organizing function of living systems which is connected and interacting with the body, which plays a more structural role in living systems [10]. Additionally, the concept of health is shifting back to interpretations that are more comprehensive. For instance, the concept of salutogenesis defines health 


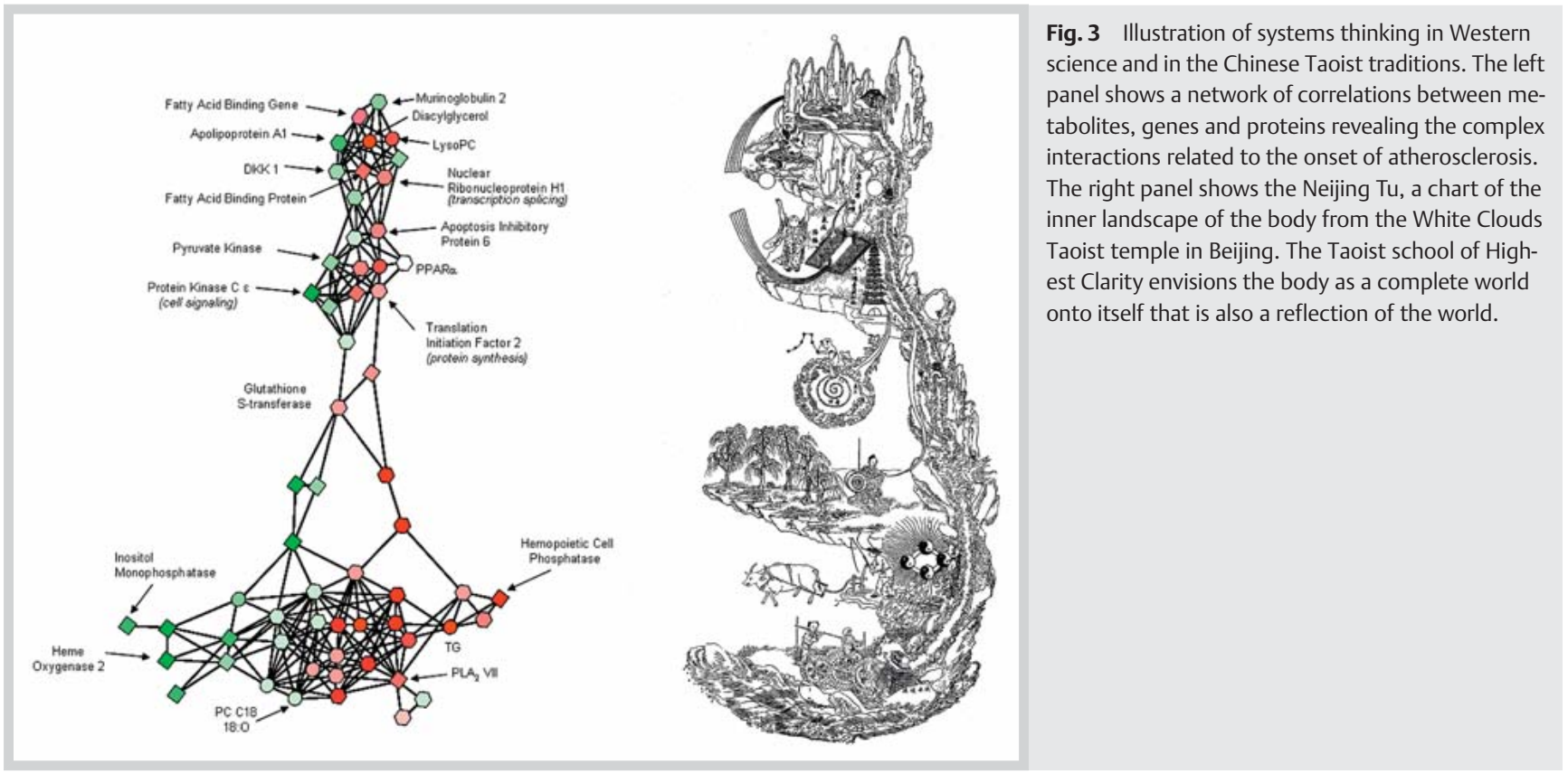

in terms of movement and connection [11].The capacity to move in a health-promoting direction is determined by comprehensibility, meaningfulness and manageability [12].

The systems approach to medicine that is now developing in the West has the potential to integrate with Chinese medicine [13]. In particular, the systems biology approach of patient profiling using modern genomics, proteomics and metabolomics technologies $[14,15]$ is a perfect match for the systems diagnosis in Chinese medicine. Integration of these approaches may reveal different groupings or subphenotypes of patients, which require different treatments. Additionally, knowledge about the biological mechanisms behind the personalized herbal formulas used in Chinese medicine is expanding through modern herbal chemical profiling techniques $[16,17]$.

- Fig. 3 illustrates the Western systems biology view of the body and the Chinese view of the body as a landscape [15]. The correlation network in the left panel shows the relationships between metabolites, proteins and genes during an early stage of atherosclerosis. In the right panel, a drawing illustrates the relationships within the body. By zooming in elements can be found that have a relationship with Western perspectives. For example, the fire in the cauldron in the lower part in 0 Fig. 3 is called MingMen (the gate of life), which can be compared to the adrenal glands. MingMen catalyzes and supports processes in the body, such as temperature and metabolism, which is similar to adrenal hormone function.

Both Chinese and Western science describe life as a complex, dynamic, non-linear system. As Western scientists discovered the non-linear behaviour of cytokine networks, Chinese practitioners have recognized non-linear patterns in how symptoms change in patients [18]. The fractal properties of the arteries, lungs and heart rate resemble the fractal thinking in Chinese medicine [19]. In both sciences, life is considered a self-organizing system that is far from equilibrium. Systems thinking can build the cultural, philosophical and scientific bridges that are necessary to share understanding between the two sciences. By studying tools and techniques developed in both the Chinese and Western medical systems, new insights will emerge that are nec- essary to heal the patient, his environment, and the world he lives in.

Both Western systems biology and Chinese Taoist science consider the complete body and its relationships and connections to its environment. These similarities bring the two sciences closer together. Essentially, the world inside and outside the body are the same. Mapping relationship patterns has become an important strategy in modern systems biology research. For instance, advanced "-omics" technologies can be used to reveal diagnostic insights or indicate the impact of interventions [14].

Traditionally, the focus of the systems view in Chinese medicine has been directed towards health promotion. In recent Western terminology, this is known as strengthening the resilience of the homeostatic process, or salutogenesis. Typically, Western medicine has focused on disease management.

- Fig. 4 illustrates a healthy dynamic situation that loses its control capability (homeostasis) and develops into a disease state [20]. The schematic is oversimplified, since the border between health and disease depends on the individual and may not always be clear. Moreover, the process is multidimensional, so the twodimensional representation is limited for evaluation. The chart represents a typical readout if the diagnostic tool for monitoring health is based on a reductionist principle, such as blood pressure or glucose level measurements. When the system is incapable of maintaining health, a disease state occurs. In this scheme, a reductionist intervention $\left({ }^{*} 1\right)$ can be applied to reduce the given symptom. While the reductionist intervention may appear promising, when the medication is stopped the system cannot maintain control, and the disease state recurs [21]. In contrast, application of a system treatment $\left({ }^{*} 2\right)$ allows the system to regain its resilience. Eventually, when the treatment is stopped, the system remains within the healthy range. Instead of monitoring a single variable, use of a system dynamics approach is more effective, which is noted especially in chronic conditions or during prevention strategies. In acute states, reductionist approaches can be very effective for releasing the immediate danger; however, strengthening the system provides a powerful additive value. For instance, during chemotherapy, Chinese medi- 


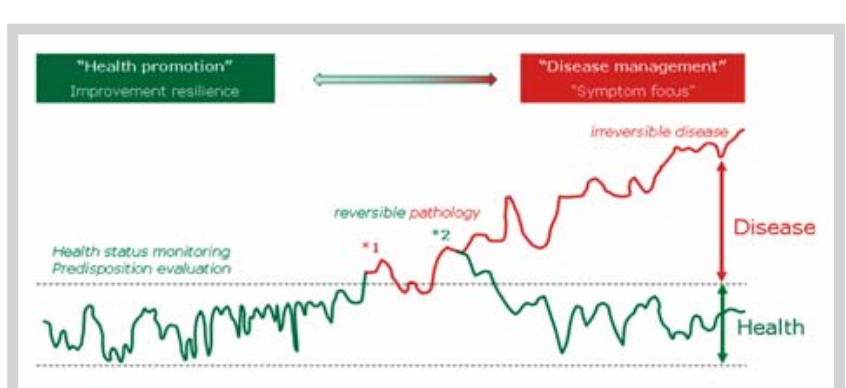

Fig. 4 The schematic representation of a dynamic system losing its homeostasis and developing into a disease state. The effects of $\left({ }^{*} 1\right)$ a reductionist intervention and $\left({ }^{*} 2\right)$ a system intervention are illustrated.

cine can protect or reduce potential side effects and strengthen immune function.

Therefore, 0 Fig. 4 illustrates the bridge between Chinese and Western medicine shown in 0 Fig. 1 from a different angle. In O Fig. 4, major emphasis from Western medicine is on disease management while the major emphasis on health promotion comes from Chinese medicine.

\section{System-Based Diagnosis, Building the Bridge \\ $\nabla$}

Today, the term diagnosis has a wide variety of meanings depending on its context. The word originated from a combination of the Greek words "dia-", meaning "apart" or "split" and "gnosi", meaning "to learn" or "knowledge". Therefore, a diagnosis is related to a recognizable separation (or split) from the whole. In a medical context, a diagnosis is typically used to describe a certain condition or syndrome that is characterized by a combination of specific features that occur simultaneously and vary from "normal" functioning. A diagnosis can lead to the recognition of a specific disease or syndrome based on a group of symptoms or signs that characterize an "abnormal" condition. In Greek, the word "symptom" refers to a feeling of misfortune that has befallen a patient. The experience of confronting disease symptoms varies substantially in different cultures and in people with different world views, as discussed below. A differentiation can be made between symptoms that are described by the patient (subjective) and signs that are observed or measured by others (objective). For instance, a symptom could include pain sensations, and a sign could include glucose or cholesterol levels.

From a system view, there is an implicit danger while making a diagnosis because by characterizing a person using a selection of symptoms and signs and emphasizing the difference from what is believed to be normal or healthy, there is a loss of context information. As mentioned previously, this is one of the major differences between Chinese and Western medicine. In Western medicine, the basis for developing therapeutic interventions often involves reducing a generic observation of a condition to the lowest number of possible combinations of symptoms and signs. For instance, type 2 diabetes is diagnosed by one sign, a person's glucose levels. As such, Western medicine has become successful in reducing symptomatic effects, which is relevant in acute or relatively threatening conditions. Corrective treatments focus on specific aspects rather than stimulating or guiding endogenous (homeostatic) regulatory processes, which is typical in systemsbased approaches. The reductionist approach aims for simplified diagnosis-therapy protocols that have become the basis for quality management in health care.

This focus has advantages, but it also has clear limitations, which are evident in patients with chronic diseases or in situations requiring personalized medical approaches. Previously, these limitations were discussed from a systems perspective for drug discovery and drug development [22] and from a personalized medicine perspective [23]. At present, a clear diagnostic differentiation combined with a specific therapy has become a new driving force known as companion diagnostics or theranostics. This approach led to improvements in specific situations, such as cancer therapy, but it still follows a reductionist path as its basis. This trend will certainly continue in the coming years, and we will discuss this in detail below. However, to understand the limitations of this process and to reveal the unaddressed potential in these cases, we will first examine the diagnosis process in more detail. A general schematic illustrating patient-provider interactions from a system's view can serve as the basis for understanding a number of important issues ( $\mathbf{O}$ Fig. $\mathbf{5}$ ). The first step in the diagnosis process is recognition by a person that his/her ability to function in daily life is limited or challenged. These limitations may include a variety of physical and psychological features. In fact, a subjective shift in consciousness has already emerged that something is wrong, which is often followed by a feeling of fear that the condition will deteriorate in the future. Our thinking patterns often gravitate towards envisioning a future that is different from what we think is desired or essential (thinking based on preconceived ideas), and this is a first step towards becoming unhappy and stressed [24]. The next step is typically an appointment with a general practitioner. During the appointment, which is schematically depicted in $\boldsymbol{O}$ Fig. 5 , many things happen simultaneously that have distinct influences on the diagnostic process. O Fig. 5 reflects the essence of the meeting between the patient and physician and the importance of the connectivity for exchanging information. Typically, a physician will ask questions or invite the patient to explain his or her motivation for visiting. In the mean time, body language and intuitive processes are active. The process of anamnesis has begun, and the physician collects information that is sometimes combined with a physical examination, during which symptoms and signs are gathered. It is important to note that by asking questions, the consciousness and the awareness of the patient is already influenced and changed. Therefore, therapy and personal awareness has already begun.

The information collected relates not only to the patient's status at that point in time in isolation, but also to the person's social network, culture and history. These latter aspects are of key importance in the systemic view to fully understanding the patient. In fact, we should consider a biosphere that includes both the patient and the physician $[25,26]$. The physicians' biosphere must have the ability to communicate and the world view (spirituality) of the patient and physician play a major role in this process. Although not the focus of this paper, we would like to emphasize here that the diagnostic process will follow different routes depending on different world views. For instance, when the belief is that a "misfortune" happened to the patient, this then leads to an intervention (physician-driven, corrective action). In contrast, if the situation is viewed as an "exploration" where decisions are made together about what the next step should be in the emerging future, then the patient accepts responsibility for his consciousness (reflective and process orientated). Here the interaction is reflective and process orientated, and the physician acts more like a coach. 


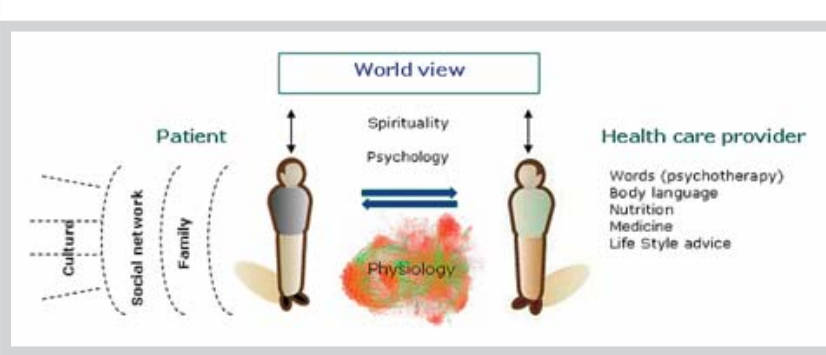

Fig. 5 Schematic illustration of the interaction between a patient and a health care provider. The quality of the connection is of key importance for the diagnostic and therapeutic process.

In the anamnesis process in Western medicine, the physician provides direct advice to the patient or refers the patient to a medical specialist for a more detailed investigation. This process leads towards more reductionism in many cases. Additionally, the physician may prescribe or suggest an intervention with medicine. A clear shift has taken place in recent years, and medical advice has begun to comprise medicine along with suggestions for lifestyle changes, such as fitness, mental training and nutrition. This reflects a shift towards a more systemic approach, recalling the original Greek definition of the word "anamnesis," which means "remembering who you are" (in context of the whole).

Let us now examine how a broader diagnostic view can cause shifts in the development of modern medicine. Modern Western medicine is based on a reductionist view. It involves studying aspects or portions of physiology, while ignoring most of the influences of psychology or spirituality, with the exception of psychiatry.

In recent decades, this way of thinking has become more balanced. Much of this change in perception is due to placebo research. The placebo effect has been heavily debated for many years. The effect is typically considered as an annoyance in scientific medical research, since it hampers the ability to analyze the "pure" pharmacodynamic effects of a drug under development. The expression, "placebo effect," reflects the feeling that it is something unscientific and without value.

Even in surgical studies, placebo effects have been reported. Pain reduction experiments with "hidden treatments," demonstrated that the placebo effect is much smaller when patients were unaware that they are receiving medication. In depression research, placebo effects can account for at least $75 \%$ of the observed positive effects of medications $[27,28]$. According to Wayne Jonas (Placebo Symposium, Nov. 27-28, 2009, Starnberg), placebo effects are influenced by: (1) pill colour, (2) number of pills or treatments, (3) compliance with treatment, (4) packet label, (5) form of treatment (pill, needle, heat, injection, laser), (6) location of the treatment (home, hospital or optimal healing environment [OHE]) (7) order or administration of treatments, (8) tone of treatment delivery and the authority of the physician, (9) information provided with delivery, (10) inherent social and cultural factors, (11) effectiveness of the drug, (12) effectiveness of other drugs with similar uses, and (13) combinations of the above factors.

In summary, placebo responses are observed frequently in clinical trials, and they vary considerably $[29,30]$. Therefore, it can be difficult to prove that the effectiveness of a drug is superior to a placebo. When a cause or variable has such a strong effect on the clinical trials for developing medicines, the factor should be extensively studied. For the placebo effect, this is especially impor- tant, since the effect significantly affects both diagnostic and therapeutic medical practices. Additionally, a reverse placebo effect called the "nocebo effect" (Latin for "I will harm," and named by Walter Kennedy in 1961) has also been reported. The nocebo effect indicates a pessimistic view of a medicine under investigation.

In a recent placebo workshop (Starnberg 2009, Nov. 27-28, 2009), Jonas argued that "for a better understanding we need to disentangle the 'placebo effect' as the physiological, psychological and clinical effects of meaning and context (MAC) and learning" [31]. This is mandatory before taking the next step towards a systemic approach in health care.

To understand the placebo effect, scientists must separately study the effect of the placebo substance (e.g. sugar) and the effect of administering the placebo (psychological responses induced by the health care environment). Often the natural cause of a disease is also taken into account. From a systemic perspective, this encompasses the movement of the whole, including psychological and spiritual factors. At this point, it is important to recall the split between body and mind in Western sciences, which was introduced by Descartes. This split led to the design of scientific experiments to reveal "pure" medication effects, which excluded the possible effect of the observer. This concept is now changing as the systemic view has emerged.

It is beyond the scope of this paper to go into further detail on placebo effects. We recommend that the interested reader consult the following authors as an entry to the placebo field: Fabrizio, Wallach, Kolls and Jonas. Additionally, we recommend exploring the references cited in the following papers [29,32-38].

Following diagnosis, the relationship between the patient and the physician is crucial. The person's world view, including their family, social network, culture, and history, are necessary for context, as shown in 0 Fig. 5. Furthermore, the physiological network depicted in the figure is a reflection of the system response to all of these effects. In systems biology studies, this network is not always appreciated, and sources of unknown variation are not linked to other important effects. The abilities of a physician to connect to the world of the patient and use intuition and authority are extremely important. Despite the efficiency and costcontrol pressures on medical health care systems today, excellent physicians are still able to use these skills. In modern medical education systems, relatively little, if any, attention is aimed at understanding the whole patient.

What does this mean for diagnostic strategies? From $\bullet$ Fig. 5, it is clear that from a systems view it is essential to consider the interactions of the person with his environment as a whole. The diagnosis is a description of a person at a given point in time, knowing the person's history and addressing his current condition, which is expressed as a disease. People with different cultural backgrounds will have different views, and consequently have different needs. The aim of the diagnosis is to find a way to support a person by relieving him from disabling factors or making him aware of necessary lifestyle changes, including changes in psychological thinking patterns.

In Chinese medicine, diagnosis is highly related to the above contextual description. A person is seen as unique, but also as part of a whole. Both the patient and the doctor grow during their interaction in their knowledge of life and its purpose. The modern technologies developed in Western medicine have become valuable for obtaining a deep insight into the physiological mechanisms of disease. Chinese medicine can be enriched enormously by adding this wealth of new diagnostic aspects. The systems bi- 
ology technology has allowed for an additional bridge between the "seen and the unseen." In fact, the current situation is rich in opportunities. Descriptive diagnosis can be linked to a systemsbased medical system. Additionally, technological capabilities have opened up new insights into biology. The next step in modern medicine will form from a combination of both strategies in a synergy of global knowledge.

Taking this step requires that health care systems offer solutions that include patient participation. Whenever possible, the patient should remain responsible for his own health, and the health care process should follow a counselling trajectory. In a way, it can be compared to the modern view of how organizational changes can be guided through a deeper meaning (identity, soul or source of the organization) and not via a shortcut to a solution. The ultimate connection and quality of the relationship between those involved in the process is highly relevant, and a deeper understanding arises from reflection upon the whole. This approach, called "presencing," is expressed with other current management insights, such as Theory U [39]. These theories link to spiritual literature, such as the Chinese books of wisdom, the Tao Te Ching (The Book of the Way and its Virtue). Spiritual books are a source of inspiration, and the timelessness of the wisdom serves to help us discover ourselves and the purpose and meaning of our life. For instance, Lao Tzu (604-531 BC) said "If you do not change direction, you may end up where you are heading." This expression can be applied to health care as a reason to investigate current practice and envision the future. This will allow practical steps to be designed in a direction originating from current health care systems.

From a Western perspective, systems biology research can improve the "one-drug-fits-all" interventions based on reductionist diagnoses. In 0 Fig. $\mathbf{6}$, this is shown as the first step towards a more targeted approach in which subtype classes are recognized. Still the basis for this approach is disease-orientated; however, current medicines can be optimized for a better efficacy/safety ratio for a given subtype. The last and largest step towards personalized health care is diagnosis support to provide a patientcentred wellness approach. The health care ecosystem must fully utilize this potential for personalized medicine. To do so, a major driving force could be the combination therapy strategy outlined previously [40]. Chinese medicine is already based on a personalized approach, so improvements to this system must come from refining and expanding the current Western medicine options, focusing on quality control and providing scientific evidence to create a global acceptance of its practices outside Chinese culture.
In Western medicine, the step to improved subtyping (targeted care) can be strongly enhanced using knowledge from Chinese medicine. Currently, many studies are underway to detect subtypes by profiling cohorts with "-omics" technologies and systems biology platforms [14], but these evaluations must be based on non-supervised procedures or drug-response profiling for specific drugs. Gathering data from these methods is limited, since individual variation is high, as outlined above. Consequently, Chinese medicine is an attractive alternative to subtype cohorts diagnosed using Western diagnosis methods. For instance, rheumatoid arthritis (RA) or type 2 diabetes cohorts could be subtyped using pattern recognition techniques based on systems biology. This allows a subset of variables to be obtained and validated, which can serve as a new diagnostic principle when developing interventions. This strategy is the basis of the SinoDutch Centre for Preventive and Personalized Medicine. In the following example of subtyping in RA, we will demonstrate the strategy and describe the challenges in incorporating this method in practice.

\section{Towards Personalized Medicine for Rheumatoid Arthritis $\nabla$}

RA is the most common chronic, inflammatory joint disease, affecting approximately $0.5-1 \%$ of the population worldwide [ 41 , $42]$. The currently favoured treatment strategy focuses on reducing inflammation, and pain is generally believed to have a favourable effect on the disease course. However, assessments of disease activity and functional disability in patients do not always support this treatment strategy [43]. In addition, there is a high variability in treatment responses [44]. For instance, nearly $30 \%$ of patients fail to respond positively to anti-TNF therapy, which is currently the most effective therapy for RA $[45,46]$.

Predicting a patient's response to a treatment can help ensure that he receives the right treatment and minimizes the risk of side effects. Over the years, several clinical features and molecular markers have been identified that allow RA patients to be divided into subgroups that may respond differently to treatments $[47,48]$. For example, van der Helm-van Mil and colleagues demonstrated that patients who were positive or negative for anticitrullinated protein antibodies (ACPA) had distinctive RA risk profiles [49]. ACPA-positive RA patients had more inflamed joints and a higher level of joint destruction [50]. Van der Pouw Kraan and colleagues observed a large heterogeneity between the high and low INF-1 subtypes of RA patients in their gene expression

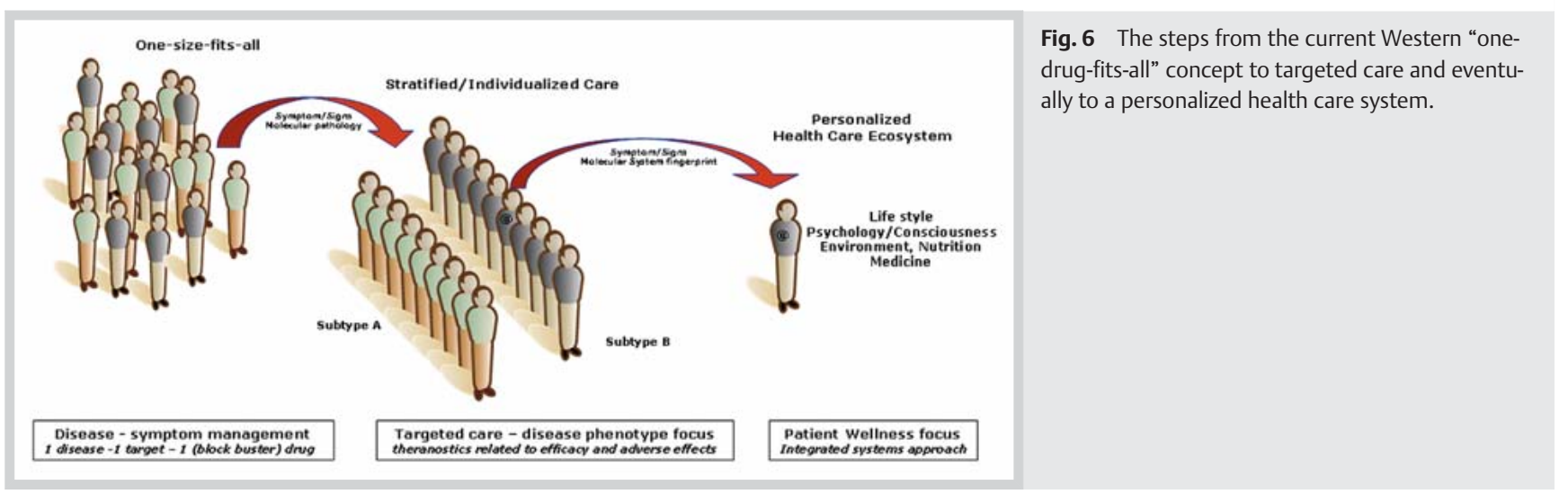


profiles, but there was no clear difference between groups in terms of clinical features [51]. Unfortunately, the knowledge gained from these studies has not yet led to personalized health care strategies in clinical practice.

Since routine molecular markers and disease activity measurements have failed to predict treatment responses, patient phenotypes should be measured more comprehensively. Glocker and others advocate use of a systems biology approach to develop personalized medicine for RA [44,52]. This type of approach would simultaneously measure the interactions between genes, proteins, metabolites and symptoms $[22,23,40]$. The resulting patient profiles can then be used to diagnose and classify patients.

Interestingly, there are several health and medical traditions that use a systems approach, including Chinese medicine. A key aspect of Chinese medical diagnosis is that symptoms are collected from the patient by the practitioner through inquiry. The symptoms and signs deigned important for determining the correct treatment vary widely from the Western perspective. To diagnose RA, rheumatologists use seven criteria established by the American College of Rheumatology [53]. These criteria involve morning stiffness, arthritis of three or more joints, arthritis of hand joints, symmetrical arthritis, rheumatoid nodules, serum rheumatoid factor and radiographic changes of the joints. In contrast, the symptoms involved in the Chinese diagnosis include climate factors, emotional states, joint problems, types of pain, fever, swellings, digestive problems, and many others [54,55]. The Chinese diagnosis provides a more complete description of the entire body.

A second advantage in Chinese medicine is that its treatment strategies can be considered a systems biology approach. For thousands of years patients in China have been treated with personalized herbal formulas based on extensive diagnoses. Herbal formulas contain hundreds of compounds of which the functions are often unknown. Interestingly, the combinations of compounds in herbal formulas work together to improve effectiveness and reduce side effects [13]. Additionally, an optimization strategy is often followed in which patients receive different or adapted herbal formulas after each consecutive consultation to improve the effectiveness or change the treatment target.

The differences in the health, disease and treatment perspectives between Western doctors and Chinese experts makes it difficult to simply transfer herbal treatments to the Western medical practice. Several herbal treatments for RA have been studied with ambiguous results because important subtype information was ignored. For example, Tripterygium wilfordii Hook. F. extracts are commonly and successfully used as a Chinese medicine intervention strategy for RA, and the extract has been studied extensively $[56,57]$. Preparations of Ganoderma lucidum (Leyss. Ex Fr.) Karst, an extract of multiple herbs called San-Miao-Wan [58], Celastrus aculeatus Merr. [59], and Forsythia suspense (Thunb.) Vahl [60] have been used as immune modulators in RA patients. In these studies, the benefits of using standard Chinese medicine preparations in RA patients were not always clear. We believe that it is necessary to develop tools to determine which subgroups of patients will benefit from specific Chinese medicines before they can be effectively introduced into Western clinical practice. The key issue here is diagnosis.

In a study of 396 RA patients, a combination of Tripterygium wilfordii Hook. F. with a Yishen Juanbi tablet was effective in patients affected with joint pain and joint tenderness, who did not experience increased urination at night and joint stiffness [61]. Addi- tionally a Western treatment consisting of diclofenac, methotrexate and sulfasalazine was found to be more effective in RA patients displaying joint tenderness and thirst, and less effective in RA patients with dizziness [61]. These results indicate that a Chinese medical diagnosis can be used to identify RA subtypes. Specifically, Chinese medical diagnoses may identify RA patient subtypes that have different responses to Western treatments or require different Chinese herbal treatments.

The next section explores the Chinese medical diagnosis of RA patients in further detail. In Chinese medicine theory, RA is part of a group of syndromes, called the "Bi-syndromes". The character "Bi" means blockage or obstruction. In Chinese medicine, this term is used to denote "obstruction of Qi and blood in the channels and collaterals" [54]. These obstructions mainly lead to pain, numbness and stiffness. The "Bi-syndromes" include other Western diseases, such as osteoarthritis, frozen shoulder, repetitive strain injuries (RSI), other rheumatic diseases, such as fibromyalgia and systemic lupus erythematosus, as well as conditions like bursitis and synovitis [54].

"Bi-syndromes" are caused by an attack of three out of four external pathogenic factors called "Heat", "Cold", "Dampness" and "Wind". After its first superficial stage, the disease can develop into later stages that can be classified according to the affected "Fu organs" or the affected "Five Tissues" [7]. A Chinese medicinal expert can determine the type of "Bi-syndrome" a patient is suffering from by employing diagnostic methods, such as inquiry, palpation, taking the pulse and looking at the tongue. Symptoms and signs related to the "Bi-syndromes" are collected, resulting in a pattern of relationships that can be interpreted and treated.

We can think about the patterns of symptoms and the relationships between symptoms and syndromes in terms of Western network theories and visualization methods. In $\odot$ Fig. 7, the patterns of symptoms and syndromes that make up the "Bi-syndromes" are visualized using Cytoscape [63]. It is immediately clear that the "Bi-syndromes" (red hexagons) are intricately related and connected by certain specific symptoms (yellow circles). In fact, the syndromes act as nodes within the dynamic network of symptoms. While a single symptom, such as dry mouth, does not point toward a syndrome, a combination of symptoms, such as dry mouth, a warm feeling and an aversion to heat, suggest that the patient has a "Heat syndrome". Some symptoms are related to multiple "Bi-syndromes" and can indicate a change in the disease from one state to another. These symptoms can be referred to as bridge symptoms or bifurcation points. For example, a patient with side pain, thirst, headache and cold feet over time could develop a new symptom like lower back pain. This indicates that the disease might suddenly shift from one stable state to another. In this case, the shift is from a liver disorder to a kidney problem, which reflects a more chronic stage of the disease.

Although the Western concept of RA is very different from the "Bi-syndromes", there are some connections between the two disease classifications. We compared the symptoms and signs of RA described in the Merck manual with those related to the "Bisyndromes" [64]. Only four symptoms were shared in both classifications: stiff joints, swollen joints, fever and pain with redness and swelling. Additionally, we compared the symptoms and signs of other rheumatic diseases with the "Bi-syndrome" symptoms. Again, there were few overlapping symptoms. $\bullet$ Fig. 8 shows the symptoms and signs that connect the "Bi-syndromes" (red hexagons) with RA and osteoarthritis (OA) (blue hexagons). Additionally, the symptoms connecting the various "Bi-syndromes" are 


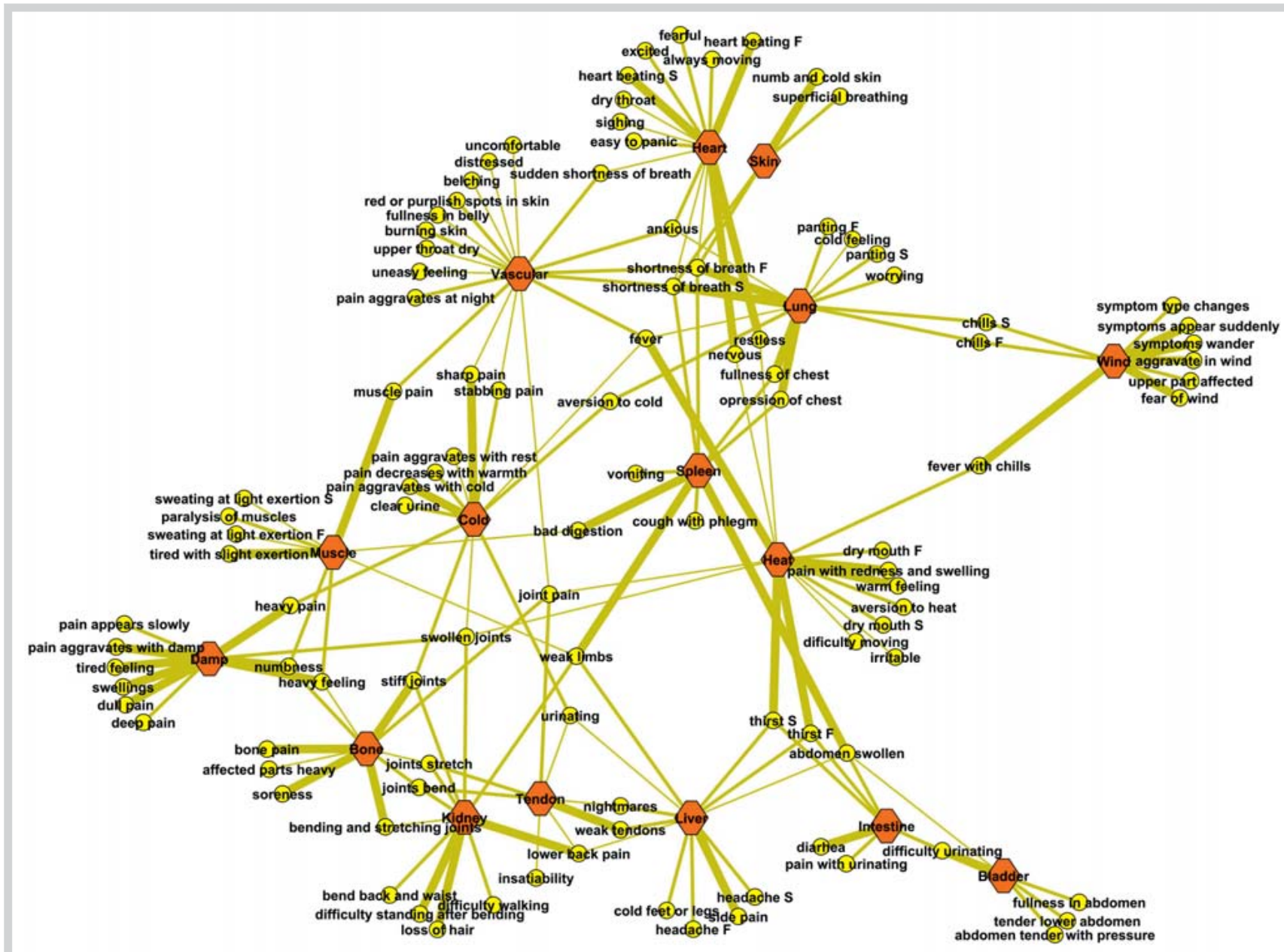

Fig. 7 Network visualization of the "Bi-syndromes" based on Vangermeersch [54]. The Cytoscape network algorithm was used to calculate a network view where the length of the edges were minimized [62]. The red hexagonal nodes represent the "Bi-syndromes", and the yellow circles represent the symptoms and signs related to the "Bi-syndromes". The green lines (edges) represent the relationships between symptoms and syndromes, and thicker lines represent stronger relationships. drawn, and the symptoms connecting RA with OA are depicted. For simplicity, the symptoms unique to single diseases were left out of the network. In Chinese medical theory, all the symptoms that are related to the "Bi-syndromes" can occur in RA patients and in patients with other rheumatic diseases. However, it proves to be difficult to match the symptoms and signs used in Chinese and Western medicine. For instance, only a few of the symptoms and signs described in Chinese medicine as being related to arthritis were listed in the Merck manual [64]. The additional symptoms and signs recognized in Chinese medicine might point towards patient subtypes.

To test our hypothesis that a Chinese medicine diagnosis could point towards RA patient subtypes that can be interpreted with modern Western systems biology techniques, we designed a study that included 33 RA patients and 16 healthy volunteers. The patients were classified into "Cold" or "Heat" groups by a Chinese medicine expert, according to Chinese medical diagnosis. All patients had swollen and painful joints at the time of diagnosis and during collection of the blood samples. The blood samples were used to perform a gene expression analysis in $\mathrm{CD}^{+} \mathrm{T}$ cells and for a broad-spectrum gas chromatography-mass spectrometry (GC-MS) metabolomics analysis [65].
"Cold" and "Heat" were specifically chosen because these Chinese medical classifications are commonly used in Chinese medical schools. Therefore, there is little controversy over how to classify patients into the two categories although patients can express both "Cold" and "Heat" symptoms at the same time. According to Zhang and colleagues, approximately half of the RA patients in the general population fall into the "Cold" type while approximately $16 \%$ are classified as the "Heat" type [66]. We also selected these two groups because there are different Chinese herbal formulas for curing RA Cold and RA Heat patients. In this study, clearly defined "Cold" and "Heat" RA patients were asked to participate.

The "Cold" pattern can be described by severe pain in a joint or muscle that limits the range of comfortable movement with pain that does not move to other locations. The pain is relieved by applying warmth to the affected area, but increases with exposure to the cold. Loose stools are characteristic of this pattern, as well as clear profuse urine and an absence of thirst. A thin, white tongue coating is observable, combined with a wiry and tight pulse. In contrast, the "Heat" pattern is characterized by severe pain with hot, red, swollen and inflamed joints. Pain is generally relieved by applying cold to the joints. Other symptoms include fever, thirst, a flushed face, irritability, restlessness, constipation 


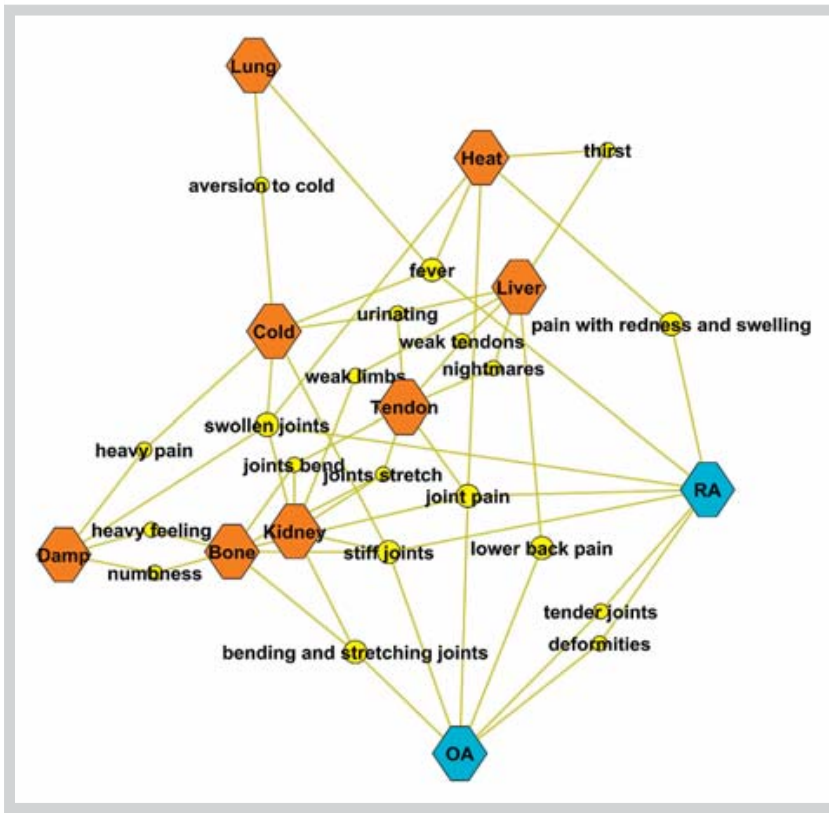

Fig. 8 Network visualization of the connections between the "Bi-syndromes" (red hexagons) and RA and OA (blue hexagons). Only the symptoms (yellow nodes) that connect a "Bi-syndrome" with a Western rheumatic disease are labelled. For simplicity, the symptoms unique to single "Bi-syndromes" or diseases were left out of the network. Although in Chinese medical theory RA and OA are connected to all the symptoms of the "Bi-syndromes", only a few of these symptoms are recognized in Western medicine.

and deep-coloured urine. The tongue may be red with a yellow coating, and the pulse may be rapid [67].

This study revealed differences in gene expression profiles and metabolomics profiles between RA Cold and Heat patients ( Fig. 9 shows the metabolomics results). These differences involved apoptosis genes, and the RA Heat group showed more activity in apoptosis-stimulating genes than the RA Cold group. In contrast, several genes related to apoptosis resistance were more active in the RA Cold group than in the RA Heat group [65]. Thus, besides finding molecular differences between the two subgroups, there is also a biological explanation using Western chemical concepts that can explain the difference in subtypes that were distinguished through Chinese medical approaches.

Apparently, the Chinese concepts of "Heat" and "Cold" are relevant in understanding RA. A more general relationship between "Cold" and "Heat" and Western medicine was well described by Li and colleagues [68]. Using a database built from PubMed articles related to keywords Heat and Cold, the researchers calculated the co-citations of these keywords with hormones, cytokines and neurotransmitters. "Heat" symptoms were related more strongly with immune factors, while "Cold" symptoms were more strongly related to hormones. Neurotransmitters were equally related to "Cold" and "Heat" symptoms [68].

It is well known that patients with autoimmune diseases, including diabetes, RA and lupus, have autonomic nervous system dysfunctions [69]. The autonomic nervous system - the sympathicus and parasympathicus - regulate basic physiological processes, such as heart rate, blood pressure, respiratory rate, gastrointestinal motility and body temperature. Czura and Tracey hypothesized that dysfunction of the cholinergic anti-inflammatory pathway may predispose some individuals to excessive inflam-

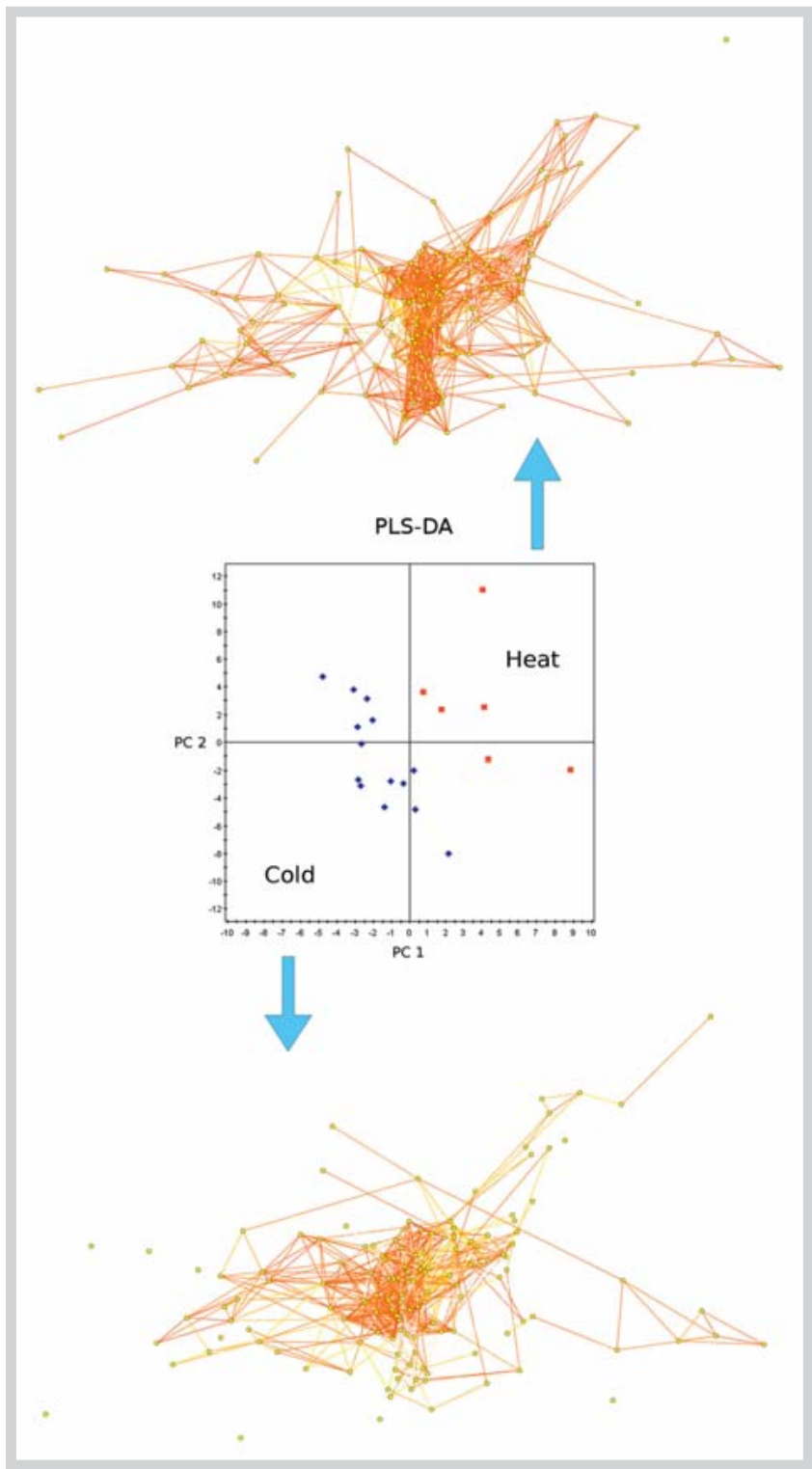

Fig. 9 The centre panel shows a partial least square-discriminant analysis (PLS-DA) score plot in which all the RA patients are represented by a dot. The blue dots represent RA Cold patients, and the red dots represent RA Heat patients. The RA Cold patients and RA Heat patients are clearly separated. The PLS-DA model was validated using cross validation and permutation testing. Above and below the score plot are network views of correlations between blood plasma metabolites measured using GC-MS in the RA patients. The top network shows correlations between metabolites $>50 \%$ for RA Heat patients, and the bottom network shows correlations between metabolites $>50 \%$ in the RA Cold patients. The nodes in both networks are the same and are composed of $>80 \%$ correlations in the RA Heat patients and $>80 \%$ correlations in the RA Cold patients. The topology of the network was generated using the Kamada algorithm, which was unweighed. The position of the nodes is equal in both networks, which means that the same metabolites (nodes) are represented in both networks, only the strength of the correlations are different.

matory responses [70]. The cholinergic anti-inflammatory pathway normally provides a brake on the immune system that restrains cytokine production. If the brake becomes insufficient, due either to insensitivity to acetylcholine released by the pathway or to diminished signals in the pathway, cytokine responses can become excessive [70]. Therefore, in RA, it is possible that 
there is both a disregulation of the autonomic nervous system and a disbalance in the immune system. Additionally, the extent to which each system is out of balance has relevance, which may be understood by studying the Chinese medicine diagnosis.

Li's study suggests that RA Cold patients suffer more from a hormone disbalance, and RA Heat patients suffer more from an immune system disbalance, although the two systems are closely related. Moreover, this suggests that RA Cold patients may respond better to a hormone treatment, such as corticosteroids, while RA Heat patients may benefit more from an immune-related treatment, such as one of the new biologicals. Although this hypothesis has not yet been tested, this could be an excellent example of subtyping RA patients using the Chinese medical diagnosis to personalize and improve the Western treatments for RA patients.

\section{Conclusion and Perspectives \\ $\checkmark$}

During the last 10 years, systems thinking has been emerging in pharmaceutical and biomedical sciences. Consequently, a natural bridge can now be formed between holistic- and reductionist-focused philosophies. There are vast opportunities available through merging the Chinese and Western medical perspectives, since both are complementary to one another and each has its own merits. For example, reductionist strategies are favourable in acute (disease management) situations, while holistic approaches offer more opportunities for preventive (health promotion) and chronic conditions.

In $\odot$ Fig. 10, an analogy of the desired situation of combining holistic and reductionist perspectives is shown as a composite photo that displays a sharp view on the whole (moon) and the details (migrating cormorants). This view reflects the desire to merge the holistic and reductionist perspectives in health care. However, to create this image, two pictures were taken in sequence, each under different focal conditions, and then the two photos were merged. Today, such an image is impossible to obtain in a single shot even with advanced cameras, but the future may reveal new options in due time.

Diagnosis will play a major role in future developments, since it will serve as a bridge between the different medical perspectives, and interventions can only be designed and exchanged based on a common diagnosis. The future points to an advanced personalized health care system in which personalized medicine is one of the key pillars. In Western medicine, the driving force is to improve the quality of life for patients as $90 \%$ of drugs work for only a limited number of patients. For instance, oncology medications have only a $35 \%$ response rate and the side effects are significant [71]. The economic resistance in leaving the blockbuster strategy has decreased, since the "one-drug-fits-all" concept is no longer a viable option. Combining subtyping and personalized medicine could bring more drugs into orphan drug status, which could be advantageous for drug development. When the efficacy/safety ratio improves, a higher return could be obtained for newly developed drugs within a smaller market. It is clear that combination therapy, the basic strategy in Chinese medicines [13] will create an important opportunity to generate the diversity needed for more personalized approaches.

Future trends in research and development will be more patientcentred, and the patient-physician relationship will be recognized more and more as a key element for providing health care in the future. Health promotion and preventive approaches must

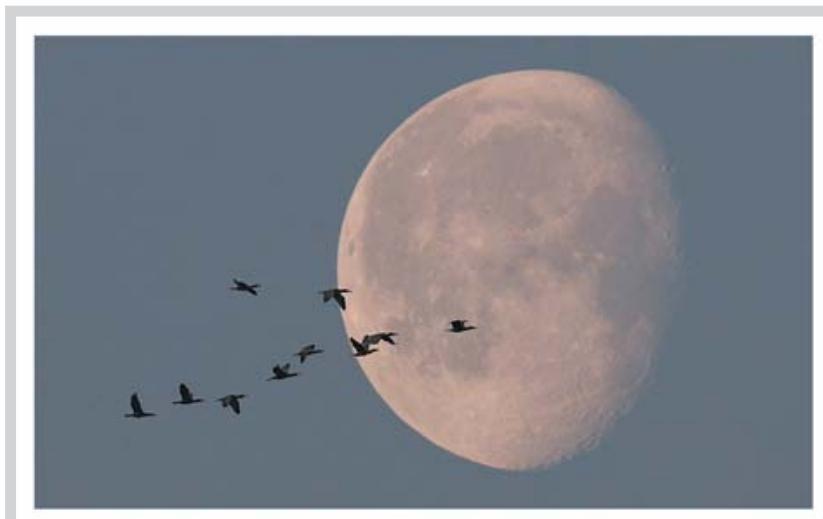

Fig. 10 A composite photo made by merging two photographs [22] taken sequentially with different focal settings. The composite photo illustrates the wish to see both the whole and the details sharply in one glance (holistic-systemic) in modern medicine.

be revised in Western medicine, since the development process is not geared towards health challenges where strengthening homeostasis is the key approach and subtle subtyping is the basis. Chinese medicine is likely to provide a variety of options based on the available diagnostic opportunities. In line with systems theory, the scale and complexity of a solution should match that of the problem. This theory indicates that combination therapy is an option, and diagnostic principles should utilize a personalized approach. Additionally, Chinese medicine can be used to strengthen the biological system, maintain a better basic health and reduce side effects. These approaches can be developed further when Chinese-based diagnostic principles are scientifically proven and translated into biochemical fingerprints.

Systems biology-based diagnostics and particularly metabolomics will be essential in this merging process, since it captures relevant phenotype information. Studying the dynamics of systems might become mandatory to provide early, more preventive diagnoses. Health care is expected to change fundamentally in the next 25 years based on these new insights and the driving force from both the quality of life and health care cost perspectives. The Sino-Dutch Centre for Personalized and Preventive Medicine is contributing to these developments by stimulating education and through scientific research that applies metabolomics-based systems biology that is guided by the principles of Chinese medicine-based diagnostics. Disease subtyping is believed to improve the understanding of responder and non-responder challenges in Western medicine, to optimize cohort selection in clinical trials, and to underpin the current understanding of the combined use of Chinese and Western medicine in China. Most importantly, subtyping will provide a scientific basis to understand and globally integrate the complementary Chinese and Western medical systems.

\section{Acknowledgements}

$\nabla$

This work was sponsored by the Netherlands Genomics Initiative, Chinese Academy of Sciences, Ministry of Science and Technology (China) (Grant No.: 2009DFA41250, 2007DFA31060, 2006BAI11B07), TNO (NL), Netherlands Metabolomics Center, The Sino-Dutch Centre for Preventive and Personalized Medicine, 
the National Genomics Initiative and the Osteo- and Rheumatoid Arthritis Foundation (NL).

\section{Affiliations}

1 Sino-Dutch Centre for Preventive and Personalized Medicine, Zeist, The Netherlands

2 TNO Quality of Life, Zeist, The Netherlands

${ }^{3}$ Leiden University, Netherlands Metabolomics Center, Division of Analytical Biosciences, Leiden, The Netherlands

${ }^{4}$ CAS Key Laboratory of Separation Science for Analytical Chemistry, Dalian Institute of Chemical Physics, Chinese Academy of Sciences, Dalian, People's Republic of China

5 SU BioMedicine, Zeist, The Netherlands

\section{References}

1 Marshall E. Science and the stimulus. Medicine under the microscope. Science 2009; 326: 1183-1185

2 Vries GD. De ontwikkeling van wetenschap: Een inleiding in de wetenschapsfilosofie. Groningen: Wolters-Noordhoff; 1984

3 Pearcy LT. Diagnosis as narrative in ancient literature. Am J Philol 1992; 113: 595-616

4 Pagel $W$. Prognosis and diagnosis: a comparison of ancient and modern medicine. J Warburg Inst 1939; 2: 382-398

5 Hua-Ching $N$. The book of changes and the unchanging truth, 2nd edition. Los Angeles: SevenStar Communications; 1999

6 Schroën J. Non-linear dynamics and Chinese medicine: an essay on research models, TCM and recent changes in modern scientific philosophy. Clin Acupunct Orient Med 2002; 3: 92-98

$7 \mathrm{Ni}$ M. The Yellow Emperor's Classic of Medicine: A New Translation of the Neijing Suwen with Commentary, 1st edition. Boston: Shambhala; 1995: 316

8 Wilber $K$. The marriage of sense and soul: integrating science and religion. Reprint. New York: Random House; 1999

9 Hahn RA, Gaines AD. Physicians of Western medicine. Berlin: Springer; 1985

10 Capra F. The hidden connections: integrating the biological, cognitive, and social dimensions of life into a science of sustainability. New York: Doubleday; 2002

11 Antonovsky A. Unraveling the mystery of health. How people manage stress and stay well. San Francisco: Jossey-Bass; 1987

12 Lindström B, Eriksson M. Salutogenesis. J Epidemiol Community Health 2005; 59: 440-442

13 Wang M, Lamers RJAN, Korthout HAAJ, van Nesselrooij JHJ, Witkamp RF, van der Heijden R, Voshol PJ, Havekes LM, Verpoorte R, van der Greef J. Metabolomics in the context of systems biology: bridging traditional Chinese medicine and molecular pharmacology. Phytother Res 2005; 19: $173-182$

14 Van der Greef J, Martin S, Juhasz P, Adourian A, Plasterer T, Verheij ER, McBurney $R N$. The art and practice of systems biology in medicine: mapping patterns of relationships. J Proteome Res 2007; 6: 1540-1559

15 Clish CB, Davidov E, Oresic M, Plasterer TN, Lavine G, Londo T, Meys $M$ Snell P, Stochaj W, Adourian A, Zhang X, Morel N, Neumann E, Verheij E, Vogels JTWE, Havekes LM, Afeyan N, Regnier F, van der Greef J, Naylor S. Integrative biological analysis of the $\mathrm{APOE}^{*} 3$-leiden transgenic mouse. OMICS 2004; 8: 3-13

16 Wang J, van der Heijden R, Spruit S, Hankemeier T, Chan K, van der Greef J, Xu G, Wang M. Quality and safety of Chinese herbal medicines guided by a systems biology perspective. J Ethnopharmacol 2009; 126: 31-41

17 Chang WT, Thissen U, Ehlert KA, Koek MM, Jellema RH, Hankemeier T, van der Greef J, Wang $M$. Effects of growth conditions and processing on Ehmannia glutinosa using fingerprint strategy. Planta Med 2006; 72: 458-467

18 Callard R, George AJ, Stark J. Cytokines, chaos, and complexity. Immunity $1999 ; 11: 507-513$

19 Higgins JP. Nonlinear systems in medicine. Yale J Biol Med 2002; 75: 247-260

20 Van der Greef J, Stroobant P, van der Heijden $R$. The role of analytical sciences in medical systems biology. Curr Opin Chem Biol 2004; 8: 559565

21 Kwee SH, Tan HH, Marsman A, Wauters C. The effect of Chinese herbal medicines (CHM) on menopausal symptoms compared to hormone replacement therapy (HRT) and placebo. Maturitas 2007; 58: 83-90
22 Van der Greef J. Systems biology, connectivity and the future of medicine. Syst Biol 2005; 152: 174-178

23 Van der Greef J, Hankemeier T, McBurney RN. Metabolomics-based systems biology and personalized medicine: moving towards $n=1 \mathrm{clinical}$ trials? Pharmacogenomics 2006; 7: 1087-1094

24 Tolle E. The power of now. Novato: New World Library; 2004

25 Wilber K. Integral medicine: a noetic reader. Available at http://wilber shambhala.com/html/misc/intergal-med.pdf. Accessed January 21, 2010

26 Lovelock JE, Margulis L. Atmospheric homeostasis by and for the biosphere - the Gaia hypothesis. Tellus 1974; 26: 2-10

27 Kirsch I, Sapirstein G. Listening to Prozac but hearing placebo: A metaanalysis of antidepressant medication. Prev Treat 1998; 1: article 0002a

28 Kirsch I, Deacon BJ, Huedo-Medina TB, Scoboria A, Moore TJ, Johnson BT. Initial severity and antidepressant benefits: a meta-analysis of data submitted to the food and drug administration. PLoS Med 2002; 5: 260-268

29 Walach H, Sadaghiani C, Dehm C, Bierman DJ. The therapeutic effect of clinical trials: understanding placebo response rates in clinical trials a secondary analysis. BMC Med Res Methodol 2005; 5: 26

30 Walach H, Falkenberg T, Fonnebo V, Lewith G, Jonas WB. Circular instead of hierarchical: methodological principles for the evaluation of complex interventions. BMC Med Res Methodol 2006; 6: 29

31 Moerman DE, Jonas WB. Deconstructing the placebo effect and finding the meaning response. Ann Intern Med 2002; 136: 471-476

32 Giordano J. Spirituality, suffering, and the self. Mind and Matter 2008; 6: 179-191

33 Kohls $N$, Walach $H$, Lewith $G$. The impact of positive and negative spiritual experiences on distress and the moderating role of mindfulness. Arch Psychol Relig 2009; 31: 357-374

34 Kohls N, Sauer S, Walach H. Facets of mindfulness - results of an online study investigating the Frieburg mindfulness inventory. Pers Individ Dif 2009; 46: 224-230

35 Benedetti F. Mechanisms of placebo and placebo-related effects across diseases and treatments. Annu Rev Pharmacol Toxicol 2008; 48: 33-60

36 Benedetti F, Lanotte M, Colloca L, Drucati A, Zibetti M, Lopiano L. Electrophysiological properties of thalamic, subthalamic and nigral neurons during the anti-parkinsonian placebo response. J Physiol 2009; 587: 3869-3883

37 Enck P, Benedetti F, Schedlowski M. New insights into the placebo and nocebo responses. Neuron Rev 2008; 59: 195-206

38 Walach H, Jonas WB. Placebo research: the evidence base for harnessing self-healing capacities. J Altern Complement Med 2004; 10 (S1): s103-s112

39 Scharmer CO. Theory U: leading from the future as it emerges. Cambridge, MA: The Society for Organizational Learning; 2007

40 Van der Greef J, McBurney RN. Innovation: Rescuing drug discovery: in vivo systems pathology and systems pharmacology. Nat Rev Drug Discov 2005; 4: 961-967

41 Symmons DPM. Epidemiology of rheumatoid arthritis: determinants of onset, persistence and outcome. Best Pract Res Clin Rheumatol 2002; 16: 707-722

42 Hochberg MC, Silman AJ, Smolen JS, Weinblatt ME, Weisman MH. Rheumatology. Mosby: Elsevier Limited; 2008

43 Welsing PMJ, Fransen J, van Riel PLCM. Is the disease course of rheumatoid arthritis becoming milder? Time trends since 1985 in an inception cohort of early rheumatoid arthritis. Arthritis Rheum 2005; 52: 2616 2624

44 Bridges SL. Personalized medicine in rheumatoid arthritis: hopes and challenges. Bull NYU Hosp Jt Dis 2007; 65: 174-177

45 Anderson IJ, Wells G, Verhoeven AC, Felson DT. Factors predicting response to treatment in rheumatoid arthritis: the importance of disease duration. Arthritis Rheum 2000; 43: 22-29

46 Smolen JS, Aletaha D. Challenges of predicting treatment response in patients with rheumatoid arthritis. Nat Clin Pract Rheumatol 2005; 1: 62-63

47 Gaston JSH. Cytokines in arthritis - the 'big numbers' move centre stage. Rheumatology (Oxford) 2008; 47: 8-12

48 Lundy SK, Sarkar S, Tesmer LA, Fox DA. Cells of the synovium in rheumatoid arthritis. T lymphocytes. Arthritis Res Ther 2007; 9: 202

49 Van der Helm-van Mil AHM, Huizinga TWJ, de Vries RRP, Toes REM Emerging patterns of risk factor make-up enable subclassification of rheumatoid arthritis. Arthritis Rheum 2007; 56: 1728-1735 
50 Van der Helm-van Mil AHM, Verpoort KN, Breedveld FC, Toes REM, Huizinga TWJ. Antibodies to citrullinated proteins and differences in clinical progression of rheumatoid arthritis. Arthritis Res Ther 2005; 7: R949-R958

51 Van der Pouw Kraan TCTM, Wijbrandts CA, van Baarsen LGM, Voskuyl AE, Rustenburg F, Baggen JM, Ibrahim SM, Fero M, Dijkmans BAC, Tak $P$, Verweij $C L$. Rheumatoid arthritis subtypes identified by genomic profiling of peripheral blood cells: assignment of a type I interferon signature in a subpopulation of patients. Ann Rheum Dis 2007; 66: 10081014

52 Glocker MO, Guthke R, Kekow J, Thiesen HJ. Rheumatoid arthritis, a complex multifactorial disease: on the way toward individualized medicine. Med Res Rev 2006; 26: 63-87

53 Arnett FC, Edworthy SM, Bloch DA, McShane DJ, Fries JF, Cooper NS, Healey LA, Kaplan SR, Liang MH, Luthra HS, Medsger TA, Mitchell DM, Neustadt DH, Pinals RS, Schaller JG, Sharp JT, Wilder RL, Hunder GG. The American Rheumatism Association 1987 revised criteria for the classification of rheumatoid arthritis. Arthritis Rheum 1988; 31: 315-324

54 Vangermeersch L. Bi-syndromes. Brussels: Satas; 1994

55 Maciocia G. The foundations of Chinese medicine: a comprehensive text for acupuncturists and herbalists, 2nd edition. Kidlington: Churchill Livingstone; 2005

56 Tao X, Younger J, Fan FZ, Wang B, Lipsky PE. Benefit of an extract of Tripterygium wilfordii Hook $\mathrm{F}$ in patients with rheumatoid arthritis: a double-blind, placebo-controlled study. Arthritis Rheum 2002; 46: 17351743

$57 \mathrm{Gu}$ WZ, Brandwein SR, Banerjee S. Inhibition of type II collagen induced arthritis in mice by an immunosuppressive extract of Tripterygium wilfordii Hook f. J Rheumatol 1992; 19: 682-688

58 Li EK, Tam L, Wong CK, Li WC, Lam CWK, Wachtel-Galor S, Benzie IFF, Bao YX, Leung PC, Tomlinson B. Safety and efficacy of Ganoderma lucidum (lingzhi) and San Miao San supplementation in patients with rheumatoid arthritis: a double-blind, randomized, placebo-controlled pilot trial. Arthritis Rheum 2007; 57: 1143-1150

59 Tong L, Moudgil K. Celastrus aculeatus Merr. suppresses the induction and progression of autoimmune arthritis by modulating immune response to heat-shock protein 65. Arthritis Res Ther 2007; 9: R70
60 Chen X, Oppenheim JJ, Howard OMZ. Chemokines and chemokine receptors as novel therapeutic targets in rheumatoid arthritis (RA): inhibitory effects of traditional Chinese medicinal components. Cell Mol Immunol 2004; 1: 336-342

61 He Y, Lu A, Zha Y, Yan X, Song Y, Zeng S, Liu W, Zhu W, Su L, Feng X, Qian X, $\mathrm{Lu} C$. Correlations between symptoms as assessed in traditional Chinese medicine (TCM) and ACR20 efficacy response: a comparison study in 396 patients with rheumatoid arthritis treated with TCM or Western medicine. J Clin Rheumatol 2007; 13: 317-321

62 Kamada T, Kawai S. An algorithm for drawing general undirected graphs. Inf Process Lett 1989; 31: 7-15

63 Viswanathan GA, Seto J, Patil S, Nudelman G, Sealfon SC. Getting started in biological pathway construction and analysis. PLoS Comput Biol 2008; 4: e16

64 Beers $M H$. The Merck manual, 18th edition. Whitehouse Station: Merck; 2006

65 Van Wietmarschen HA, Yuan K, Lu C, Gao P, Wang J, Xiao C, Yan X, Wang $M$, Schroen J, Lu A, Xu G, van der Greef J. Systems biology guided by Chinese medicine reveals new markers for subtyping rheumatoid arthritis patients. J Clin Rheumatol 2009; 15: 330-337

66 Zhang GG, Lee W, Bausell B, Lao L, Handwerger B, Berman B. Variability in the traditional Chinese medicine (TCM) diagnoses and herbal prescriptions provided by three TCM practitioners for 40 patients with rheumatoid arthritis. J Altern Complement Med 2005; 11: 415-421

67 Jiang $W$. Therapeutic wisdom in traditional Chinese medicine: a perspective from modern science. Trends Pharmacol Sci 2005; 26: 558563

68 Li S, Zhang ZQ Wu LJ, Wang YY. Understanding ZHENG in traditional Chinese medicine in the context of neuro-endocrine-immune network. IET Syst Biol 2007; 1: 51-60

69 Capellino S, Straub RH. Neuroendocrine immune pathways in chronic arthritis. Best Pract Res Clin Rheumatol 2008; 22: 285-297

70 Czura J, Tracey KJ. Autonomic neural regulation of immunity. J Intern Med 2005; 257: 156-166

71 Jackson $D B$. Clinical and economic impact of the nonresponder phenomenon - implications for systems based discovery. Drug Discov Today 2009 ; $14: 380-385$ 